\title{
Endobronchial hamartoma in a young COVID-19 symptomatic woman. Radical endoscopic treatment with a disposable bronchoscope. Case Report
}

\author{
Carmine Guarino ${ }^{1}$, Cristiano Cesaro ${ }^{1}$, Giuseppe La Cerra ${ }^{1}$, Raffaella Lucci² ${ }^{2}$, Flavio Cesaro ${ }^{3}$, \\ Enzo Zamparelli ${ }^{4}$, Gianfranco Gaglione ${ }^{5}$, Agostina Pontarelli ${ }^{5}$, Annunziata De Rosa ${ }^{5}$, Roberto Parrella ${ }^{5}$ \\ ${ }^{1}$ Unit of Bronchology, Monaldi Hospital, Azienda Ospedaliera dei Colli, Naples; ${ }^{2}$ Unit of Anesthesia and Resuscitation, \\ Cotugno Hospital, Azienda Ospedaliera dei Colli, Naples; ${ }^{3}$ Unit of Anesthesia and Resuscitation, Monaldi Hospital, \\ Azienda Ospedaliera dei Colli, Naples; ${ }^{4}$ Units of Subintensive Care Therapy, Corps G, Cotugno Hospital, Azienda \\ Ospedaliera dei Colli, Naples; ${ }^{5}$ Unit of Respiratory Infectious Disease, Cotugno Hospital Azienda Ospedaliera dei Colli, \\ Naples, Italy
}

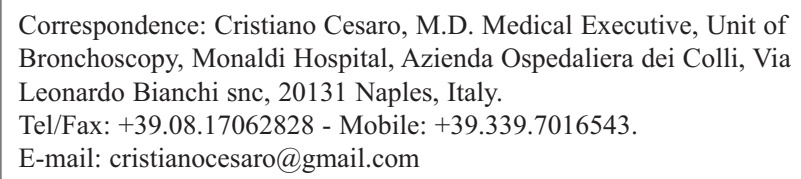

Correspondence: Cristiano Cesaro, M.D. Medical Executive, Unit of Bronchoscopy, Monaldi Hospital, Azienda Ospedaliera dei Colli, Via Leonardo Bianchi snc, 20131 Naples, Italy.

Tel/Fax: +39.08.17062828 - Mobile: +39.339.7016543.

E-mail: cristianocesaro@gmail.com

Key words: Endobronchial hamartoma; disposable bronchoscope; COVID-19; bronchoscopy; interventional pulmonology.

Contributions: CC, CG, GLC, RL, FC, EZ, GG, AP, ADR, RP, were personally involved in the case reported, had full access to all the data in the work and take responsibility for data integrity, collection, accuracy and description.; $\mathrm{CG}, \mathrm{CC}, \mathrm{FC}, \mathrm{RP}$, contributed to data collection and on the manuscript revision; CC, RL, GG, AP, ADR, RP, contributed to study design, interpretation and manuscript revision. All authors have read and approved the final version of the manuscript and agreed to be accountable for all aspects of the work.

Ethics approval: The procedure performed and described in this article was carried out in accordance with international guidelines and in accordance with the ethical standards of the Institutional Research Committee and the 1964 declaration of Helsinki and its later amendment.

Informed consent: Written informed consent was obtained from patient for publication of this case report and accompanying images.

Conflict of interest: The authors declare that they have no competing interests, and all authors confirm accuracy.

Received for publication: 26 February 2021.

Accepted for publication: 11 November 2021.

Publisher's note: All claims expressed in this article are solely those of the authors and do not necessarily represent those of their affiliated organizations, or those of the publisher, the editors and the reviewers. Any product that may be evaluated in this article or claim that may be made by its manufacturer is not guaranteed or endorsed by the publisher.

${ }^{\circ}$ Copyright: the Author(s), 2021

Licensee PAGEPress, Italy

Monaldi Archives for Chest Disease 2022; 92:1822

doi: 10.4081/monaldi.2021.1822

This article is distributed under the terms of the Creative Commons Attribution-NonCommercial International License (CC BY-NC 4.0) which permits any noncommercial use, distribution, and reproduction in any medium, provided the original author(s) and source are credited.

\begin{abstract}
Pulmonary hamartomas represent the most frequent family of benign lung tumors that typically involve the lung parenchyma and only rarely grow as endobronchial tumors. The elective treatment of endobronchial hamartoma is the bronchoscopic resection, and in those cases in which tumor extension and localization makes it not possible, surgical treatment must be evaluated. Patients with symptomatic COVID-19, hospitalized, frequently undergo a chest CT scan and in some cases, occasional findings may emerge, requiring diagnostic investigations such as bronchoscopy and interventional pulmonology procedures. Therefore, in such a delicate pathological condition, such as COVID-19, the need to perform bronchoscopy and interventional pulmonology procedures, minimizing the risk of viral transmission and ensuring necessary assistance, represents a great challenge for pulmonologists. In this article authors describe, for the first time in literature, a rare case of endobronchial hamartoma, radically resected using a single use bronchoscope, in a young female patient hospitalized for symptomatic COVID-19.
\end{abstract}

\section{Introduction}

Pulmonary hamartomas represent the most frequent family of benign lung tumors with an incidence of $0.3 \%$. Histologically characterized by fusion of different tissues originating from the embryonic mesoderm. Endobronchial hamartomas are rarer still, composing $1.4 \%$ of all pulmonary hamartomas [1]. The first choice in the treatment of endobronchial hamartoma is the bronchoscopic approach, preferably in rigid bronchoscopy. Surgical therapy, by bronchotomy or resection, should be reserved only for cases where the hamartoma cannot be approached through endoscopy, or when irreversible lung functional impairment occurred after prolonged airflow obstruction [2]. In patients with confirmed or suspected coronavirus disease (COVID-19), procedures such as bronchoscopy and interventional pulmonology procedures should be avoided, since they generate aerosols and increase the possibility of virus spreading [3]. However, if necessary, bronchoscopic procedures in COVID-19 patients should always be carried out following an organizational model and operating procedures including the use of disposable bronchoscopes, aimed at preventing contamination of endoscopic instruments and 
virus spreading to both patient and personnel involved [4-6]. Therefore, although the finding of an endobronchial hamartoma is a rather rare occurrence, in literature have been described endoscopic therapeutic approaches in flexible and rigid bronchoscopy in addition or in place of surgical resection, however, a radical endoscopic treatment using a disposable bronchoscope has never been reported.

\section{Case Report}

A 35-year-old non-smoking woman was transferred to COVID-19 the respiratory infectious disease unit from the infectious emergency unit. She was admitted with a COVID-19 positive nasopharyngeal swab complaining of fever, persistent cough, exertional dyspnea, unrelenting left-sided chest pain, and two episodes of hemoptysis reported in the preceding hours. Upon admission, arterial blood gas analysis and routine laboratory data were normal, as well as physical examination including lungs, heart, peripheral lymphnodes and abdomen. The patient underwent a high-resolution chest computed tomography scan and subsequently contrast-enhanced chest $\mathrm{CT}$ that in the right lung highlighted the presence of a neoformation, without contrast uptake, in the distal section of the intermediate bronchus with the implant base in the lower lobe bronchus (Figure 1 A-C) and downstream disventilation phenomena, no abnormalities were found in the residual lung parenchyma. Patient underwent bronchoscopy that was performed by a doctor and a nurse using a single use bronchoscope $\left(\mathrm{Ambu}^{\circledR} \mathrm{aScope} \mathrm{TM}^{\mathrm{TM}}\right.$; Figure 1D), with a probe diameter of $5.8 \mathrm{~mm}$ and a $2.8 \mathrm{~mm}$ working channel connected to the suction circuit and equipped with a AmbuaView dedicated monitor (Figure 1D) in an operating room dedicated for c COVID-19 patient, strictly in negative pressure, with depressurization flow $(\Delta$ of $10 \mathrm{~Pa})$ and unidirectional air exchange [7]. Bronchoscopy, in the portion of the intermediate bronchus bordering the right lung lower lobar bronchus, revealed the presence of a pedunculated neoformation, characterized by a smooth, reddish, poorly vascularized surface with a hard elastic consistency (Figure 1E). The inspection of the neoplasm revealed the implantation base in the medial basal segmental branch of the lower lobe bronchus, well-circumscribed and without signs of submucosal infiltration. Using a Jaw Step singleuse fenestrated crocodile biopsy forceps, introduced through the $2.8 \mathrm{~mm}$ working channel, the lesion was hooked and excised in its entirety including the implant base on the mucosa, obtaining macroscopic total removal (Figure $1 \mathrm{~F}, \mathrm{G}$ ). The procedure lasted about 15 minutes which resulted in modest bleeding that was effectively resolved by mechanical tamponade with the disposable bronchoscope. Massive bleeding was considered as a possible major complication during the procedure and therefore in the operating room setting there was a Fogarty balloon blocking catheter, such as cold physiological solution $\left(4^{\circ} \mathrm{C}\right)$, tranexamic acid and adrenaline to be used for loco-regional instillation but no immediate or late complications were recorded after procedure. The definitive histological diagnosis returned as "bronchial hamartoma" characterized by well-circumscribed, uncapsulated nodule of cartilage, respiratory epithelium and cleft of fibrous tissue (Figure 1H). Contrasted chest CT scan (Figure 1I) and bronchoscopy (Figure 1J) 18 months after treatment showed no recurrence. The patient, furthermore, was included in a six-monthly follow-up program with chest CT scan and video bronchoscopy for at least 3 years to rule out the appearance of recurrence.

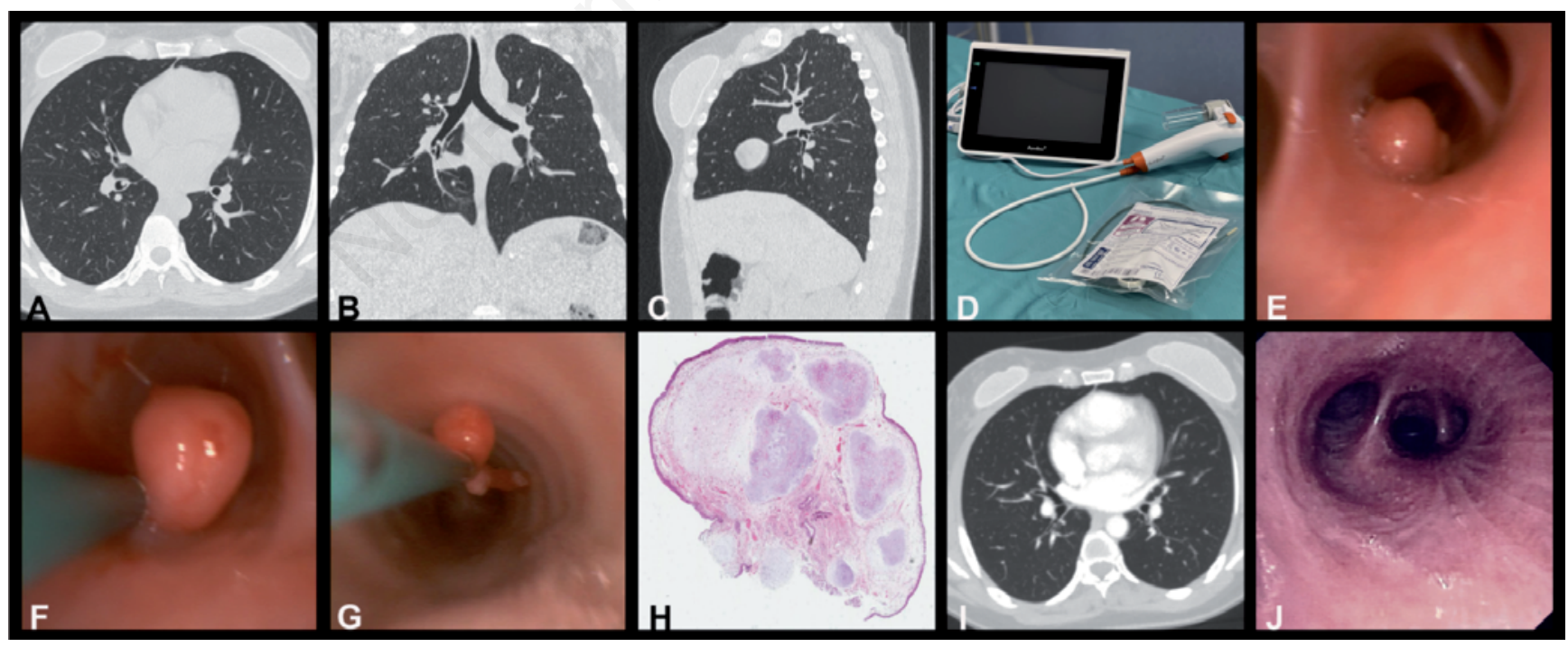

Figure 1. Axial (A), coronal (B) and sagittal (C) images of a CT scan showing neoformation, without contrast uptake, in intermediateright lower lobe bronchus. D) Image of a single use bronchoscope (Ambu ${ }^{\circledR}$ Scope $^{\mathrm{TM}}$ ) with the AmbuaView monitor and single-use biopsy forceps. E) Image of a smooth, reddish and pedunculated neoformation. F,G) Total neoformation removal with implant base using disposable biopsy forceps. $\mathrm{H}$ ) $\mathrm{H} \& \mathrm{E}$ stained image at $20 \mathrm{X}$ magnification shows an "endobronchial hamartoma" characterized by well-circumscribed and non-encapsulated cartilage nodules, respiratory epithelium and fissure of fibrous tissue. I) Axial plan of contrastenhanced CT scan 18 months after treatment, in which is highlighted the normal canalization of intermediate and right lower lobe bronchus. J) Endoscopic image 18 months after bronchoscopic treatment showing the normal canalization of the intermediate and right lower bronchus and absence of disease recurrence. 


\section{Discussion/Conclusions}

The finding of an endobronchial hamartoma is a rare event that becomes even rarer as an occasional finding in a patient hospitalized for COVID-19. Bronchoscopy is necessary to obtain the differential diagnosis and endoscopic treatment represents the GOLD standard in therapy [8]. Generally, the endoscopic approach is through rigid bronchoscopy, laser photocoagulation, mechanical resection but also electrocautery through flexible bronchoscopy may prove just as simple and effective. Surgical treatment through thoracotomy and bronchotomy is indicated only in cases where endobronchial hamartoma cannot be approached via endoscopy, or when lung resection is indicated due to the irreversible parenchyma damage from long-lasting airway obstruction $[9,10]$. In this article, for the first time in literature, the use of a disposable bronchoscope in the radical treatment of an endobronchial hamartoma has been described. The choice of disposable instruments was necessary due to the high risk of transmission of Severe Acute Respiratory Syndrome Coronavirus 2 (SARS-CoV-2) to both patients and healthcare personnel, indeed there is evidence that performing bronchoscopies in COVID-19 patients can increase virus spreading in healthcare settings. The experience acquired allows us to state that single-use bronchoscopes not only have the potential to create a safer work environment even in infectious diseases such as COVID-19, when bronchoscopy is unavoidable, and although they are currently mainly used in intensive care units for difficult intubation and to unblock bronchial secretions, they can also be considered a useful and effective tool that guarantees excellent operational functionality and a good quality of endoscopic view in the management of interventional pulmonology procedures, representing a valid alternative to multipurpose instrumentation, especially when, as in COVID-19, the use of reusable endoscopic equipment is contraindicated.

\section{References}

1. Kim JH, Jeong GM, Park KH, et al. Large endobronchial hamartoma successfully resected by snare through flexible bronchoscopy. Ann Thorac Surg 2015;100:1107-9.

2. Mondello B, Lentini S, Buda C, et al. Giant endobronchial hamartoma resected byfiberoptic bronchoscopy electrosurgical snaring. J Cardiothorac Surg 2011;6:97.

3. Verbeek JH, Rajamaki B, Ijaz S, et al. Personal protective equipment for preventing highly infectious diseases due to exposure to contaminated body fluids in healthcare staff. Cochrane Database Syst Rev 2020;4:CD011621.

4. Wahidi MM, Lamb C, Murgu S, et al. American Association for Bronchology and Interventional Pulmonology (AABIP) statement on the use of bronchoscopy and respiratory specimen collection in patients with suspected or confirmed COVID-19 infection. J Bronchology Interv Pulmonol 2020;27:e52-e54.

5. Group of Interventional Respiratory Medicine, Chinese Thoracic Society. [Expert consensus for bronchoscopy during the epidemic of 2019 novel coronavirus infection (Trial version)].[Article in Chinese]. Zhonghua Jie He He Hu XiZaZhi 2020;43:E006.

6. Pritchett MA, Oberg CL, Belanger A, et al. Society for Advanced Bronchoscopy Consensus Statement and Guidelines for bronchoscopy and airway management amid the COVID19 pandemic. J Thorac Dis 2020;12:1781-98.

7. Guarino C, Cesaro C, Fragranza F, et al. [La broncoscopia in era COVID-19. Modello organizzativo e procedure operative nell'esperienza clinica dell'A.O. dei Colli, Napoli].[Article in Italian]. Rass Patol Appar Respir 2020;35:173-8.

8. Cosio BG, Villena V, Echave-Sustaeta J, et al. Endobronchial hamartoma. Chest J 2002;122:202-5.

9. Liu C, Wang JJ, Zhu YH, Chen C. Successful use of snare electrocautery via flexible fiberoptic bronchoscopy for removal of endobronchial hamartoma causing chronic lungatelectasis and mimicking malignancy. Ther Adv Respir Dis 2017;11:435-8.

10. Abdel Hady SM, Elbastawisy SE, Hassaballa AS, Elsayed HH. Is surgical resection superior to bronchoscopic resection in patients with symptomatic endobronchial hamartoma? Interact Cardiovasc Thorac Surg 2017;24:778-82. 